\title{
Thirty-five year experience of an aggressive surgical intervention for treating post-paralytic facial synkinesis: an outcome study
}

\author{
David Chwei-Chin Chuang \\ Department of Plastic Surgery, Chang Gung Memorial Hospital, Chang Gung University, Taipei-Linkou 105, Taiwan.
}

Correspondence to: Dr. David Chwei-Chin Chuang, Department of Plastic Surgery, Chang Gung Memorial Hospital, 199 TunHwa North Road, Taipei-Linkou 105, Taiwan. E-mail: dccchuang@gmail.com

How to cite this article: Chuang DCC. Thirty-five year experience of an aggressive surgical intervention for treating post-paralytic facial synkinesis: an outcome study. Plast Aesthet Res 2021;8:5. http://dx.doi.org/10.20517/2347-9264.2020.190

Received: 13 Oct 2020 First Decision: 09 Nov 2020 Revised: 22 Nov 2020 Accepted: 1 Dec 2020 Published: 8 Jan 2021

Academic Editor: Jonathan R. Clark Copy Editor: Monica Wang Production Editor: Jing Yu

\begin{abstract}
Aim: Combined neurectomy \& myectomy and functioning free muscle transplantation is proposed as an aggressive surgical intervention for postparalytic facial synkinesis (PPFS) to effectively resolve the problem since 1985 and this treatment continues to be the standard. We aim to describe our experiences with 103 PPFS patients who underwent the surgical treatment.
\end{abstract}

Methods: A total of 103 patients with PPFS were investigated (1985-2020), but 94 were selected with all having at least one year of postoperative follow-up. Among them 50 were Type II and 44 were Type III PPFS. All patients underwent extensive removal of the synkinetic muscles and triggered facial nerve branches in the cheek, nose and neck regions, followed by gracilis transplantation for facial reanimation.

Results: The incidence of receiving the aggressive surgical intervention increased from 15\% prior to 2012 up to $24 \%$. The mean postoperative follow-up period was 10 years. Young adult (79\%) and female patients (63\%) were the dominant populations, showing their great ambition for a treatment. Results showed a significant improvement in facial smile with more teeth visible, and a significant decrease in facial synkinesis. About $96 \%$ (90 patients) did not require botulinum toxin $\mathrm{A}$ injection after surgery. Revision surgery for secondary deformity was approximately $53 \%$.

Conclusion: Treatment of PPFS is primarily reconstructive. Combined myectomy \& neurectomy and functioning free muscle transplantation for Type II and III patients are well accepted, and leads to promising and long-lasting results despite higher revision rates. Refined techniques to decrease revision rates are needed.

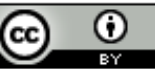

(C) The Author(s) 2021. Open Access This article is licensed under a Creative Commons Attribution 4.0 International License (https://creativecommons.org/licenses/by/4.0/), which permits unrestricted use, sharing, adaptation, distribution and reproduction in any medium or format, for any purpose, even commercially, as long as you give appropriate credit to the original author(s) and the source, provide a link to the Creative Commons license, and indicate if changes were made.

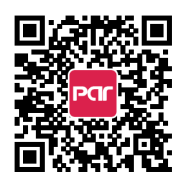


Keywords: Facial synkinesis, aggressive surgical intervention

\section{INTRODUCTION}

Pure Facial Paralysis (PFP), complete or incomplete, causes affected facial muscles paralysis (palsy) or weakness (paresis). Postparalytic facial synkinesis (PPFS), a sequela after the recovery of the PFP, complicates the involuntary and unwanted facial muscle movements or synkinesis. In PPFS there are many bizarre facial expressions, such as synkinesis between mouth and eye muscles (ocular-oral or oral-ocular synkinesis), or between mouth and neck muscles (oral-neck synkinesis) as well as many others (brow-eye, brow-mouth, mouth-platysma, chin-platysma synkinesis $)^{[1-5]}$.

With advanced micro-neuro-vascular surgery, nerve transfer and functioning free muscle transplantation (FFMT) is now becoming the mainstream surgery for long-standing facial paralysis reconstruction ${ }^{[6-11]}$. However, the treatment of PPFS remains challenging and controversial, although the mechanism of PPFS has been well studied and proposed, and the hypothesis of aberrant reinnervation is widely accepted ${ }^{[5]}$.

Several treatments have been proposed, including facial rehabilitation ${ }^{[12-14]}$, botulinum toxin A (BTX-A) injection $^{[15-17]}$ and surgical procedures ${ }^{[18-26]}$. Recommended surgical treatments for PPFS in the literature include trunk neurectomy ${ }^{[19]}$, selective neurectomy ${ }^{[2,21]}$, highly selective neurectomy ${ }^{[2,23]}$, selective myotomy or myectomy ${ }^{[24,25]}$, and combined myectomy \& neurectomy followed by FFMT $^{[26]}$. Each method has its advantages and disadvantages.

The author (DCC Chuang) started his clinical practice for facial paralysis reconstruction at Chang Gung Memorial Hospital in 1985. In the first 10 years (1985-1994) [Figure 1] conservative treatments by botulinum toxin A (BTX-A), neurectomy, myectomy, and/or rehabilitation program were performed, but were ultimately deemed impractical and insufficient. In the subsequent 25 years (1995-2020) [Figure 1] after accumulation of experience with PFP reconstruction, the author shifted the treatment to a more aggressive surgery to resolve the problem, which now becomes our standard practice. The aim of this article is to give a comprehensive and prospective review of PPFS patients who were treated by combined myectomy, neurectomy and gracilis FFMT, an aggressive surgical intervention.

\section{METHODS}

A special "Patient Examination Sheet" was designed in 1995 to record all facial paralysis patients [Supplement Digital Content 1]. The physical examination of the face is performed at repose and movement. There are five basic facial movements for recording: forehead raise, eye closure, smile with teeth exposure, snarl with lower lip pulled down, and whistle with lip pout. For PPFS, six common facial synkinesis movements induced by the individual basic movement are recorded, including frontal synkinesis with corrugator hypertrophy and tension face, eye synkinesis with eye narrowing, upper lip synkinesis with mouth commissure/upper lip twitching or elevation, lower lip synkinesis with chin skin dimples, and neck synkinesis with platysma neck bands and lower lip eversion.

Following examination with the "Patient Examination Sheet", the PPFS patients were divided into four "Types" (or Patterns) based on the quality of smile and degree of synkinesis. Quality of smile was basically dependent upon the number of teeth exposure during smiling as well as smile symmetry. Severity of synkinesis was recorded from none $(0)$, mild $(+)$, moderate $(++)$, or severe $(+++)$, based on different synkinetic movements of the face following orders of individual five basic facial expressions [Table 1 ]. 


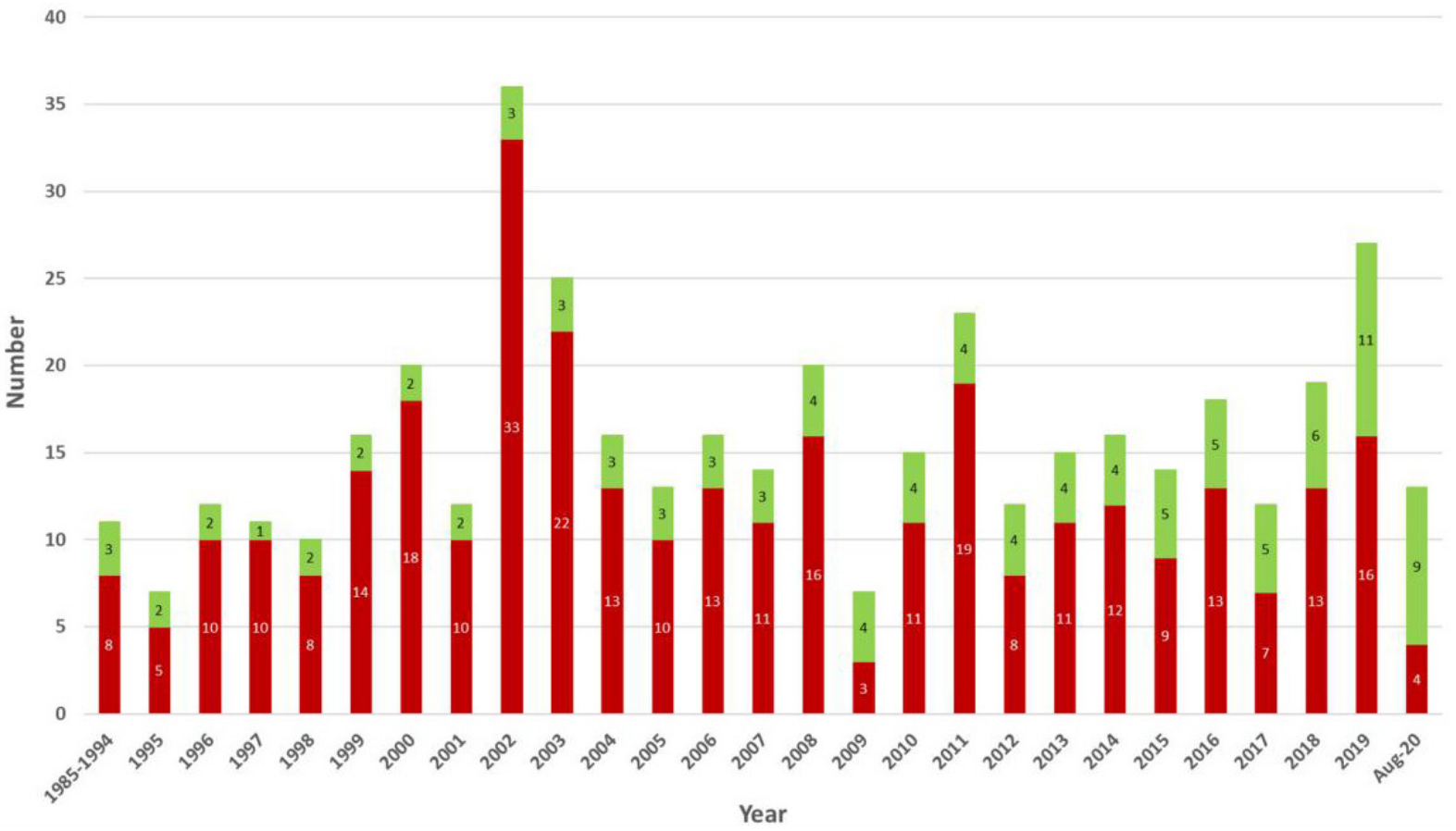

Figure 1. The year distribution of Pure Facial Palsy (PFP, red color) and Post-Paralytic Facial Synkinesis (PPFS, green color) between 1985 and 2020. Total 430 patients were treated by gracilis functioning free muscle transplantation: 327 were PFP (76\%) and 103 PPFS (24\%). PFP: Pure Facial Palsy; PPFS: Post-Paralytic Facial Synkinesis

Table 1. Movement Disorders in PPFS

\begin{tabular}{|c|c|c|c|c|c|c|}
\hline $\begin{array}{l}\text { Synkinetic } \\
\text { Movement }\end{array}$ & $\begin{array}{c}\text { Frontal / } \\
\text { corrugator } \\
\text { contraction } \\
->\text { tension face }\end{array}$ & $\begin{array}{c}\text { Orbicularis oculi } \\
\text { contraction } \\
-->\text { narrow eye }\end{array}$ & $\begin{array}{c}\text { Levators of upper } \\
\text { lip contraction } \\
\text {--> mouth angle } \\
\text { twitching }\end{array}$ & $\begin{array}{c}\text { Depressors of lower } \\
\text { lip contraction } \\
-->\text { lower lip } \\
\text { retracted }\end{array}$ & $\begin{array}{c}\text { Depressors } \\
\text { of lower lip } \\
\text { contraction } \\
\text {--> chin dimples }\end{array}$ & $\begin{array}{c}\text { Platysma } \\
\text { contraction } \\
\text {-->neck bands }\end{array}$ \\
\hline \multicolumn{7}{|l|}{ Trigger Movement } \\
\hline Forehead raise & (0) to $(+++)^{*}$ & (0) to $(+++)$ & (0) to $(+++)$ & (0) to $(+++)$ & (0) to $(+++)$ & (0) to $(+++)$ \\
\hline Eye closure & (0) to $(+++)$ & (0) to $(+++)$ & (0) to $(+++)$ & (0) to $(+++)$ & (0) to $(+++)$ & (0) to $(+++)$ \\
\hline Smile & (0) to $(+++)$ & (0) to $(+++)$ & (0) to $(+++)$ & (0) to $(+++)$ & (0) to $(+++)$ & (0) to $(+++)$ \\
\hline $\begin{array}{l}\text { Lower lip pulled } \\
\text { down }\end{array}$ & $(0)$ to $(+++)$ & (0) to $(+++)$ & (0) to $(+++)$ & (0) to $(+++)$ & (0) to $(+++)$ & (0) to $(+++)$ \\
\hline $\begin{array}{l}\text { Lip pouting } \\
\text { (whistling) }\end{array}$ & (0) to $(+++)$ & (0) to $(+++)$ & (0) to $(+++)$ & (0) to $(+++)$ & (0) to $(+++)$ & $(0)$ to $(+++)$ \\
\hline
\end{tabular}

Severity of synkinesis (4 degrees): none (0), mild (1+), moderate (2+) and severe (3+)

Type I: good smile but mild synkinesis

Type I PPFS was seen in $4 \%$ of the population ${ }^{[26]}$. The patients always show at least 4 teeth with a big smile and good appearance. Mild tension on the affected side of the face at repose and intermittent perioral twitching following eye blinking are always noted. Patients usually close the eye without lagophthalmos, but the movement is slow and weak. Good smile quality is associated with less attention to synkinesis. Usually, most patients are aware of their altered facial expression, but observers including physicians might not fully recognize the problems due to their mildness. In addition, some patients are themselves not aware that they have synkinesis. Type I patients are mostly concerned more with cosmetic issues than synkinesis.

\section{Type II: acceptable smile but moderate to severe synkinesis}

Type II PPFS was seen in $48 \%$ of the population ${ }^{[26]}$. In Type II, the patient's biggest smile shows 3-2 teeth with an acceptable smile. Their bizarre facial expression sometimes requires wearing a facemask to cover up 


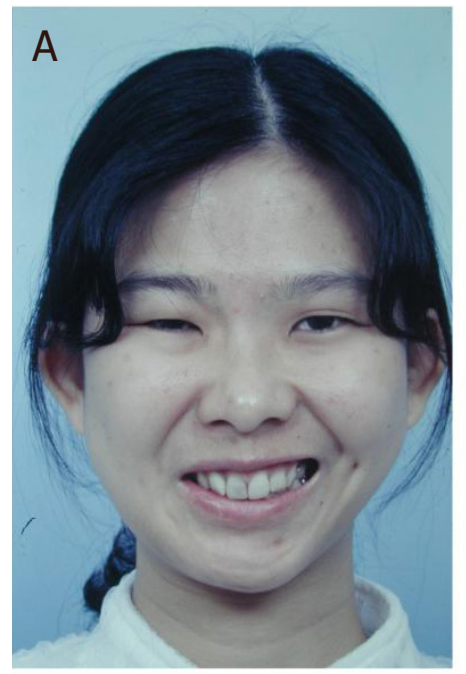

PPFS, Type II

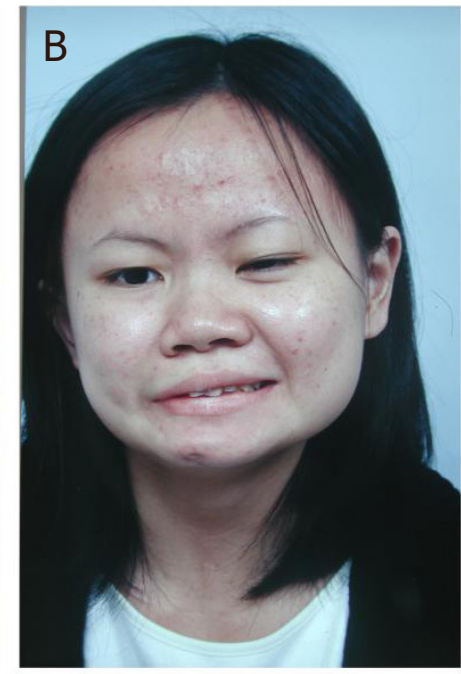

PPFS, Type III

Figure 2. Candidates of PPFS for aggressive surgical intervention: (A) patient with right PPFS Type II; (B) patient with left PPFS Type III. PPFS: postparalytic facial synkinesis

for the differences seen. Lagophthalmos with eye incompetence is often seen. The patients have moderate to severe synkinesis. Dominant narrow eye, deep nasolabial fold and dominant neck bands are commonly seen on the affected side. Some patients might have a bizarre facial expression, such as lip pouting or spitting inducing eye closure on the affected side. Facial disfigurement with synkinesis is the major reason of these patients presented for treatment [Figure $2 \mathrm{~A}$ ].

\section{Type III: unacceptable smile and moderate to severe synkinesis}

Type III PPFS was seen in $47 \%$ of the population ${ }^{[26]}$. In Type III, patients have a smile with one or no teeth visible, which leads to an unacceptable smile. Lagophthalmos is often noted. They are easily recognized given the poor smile and severe synkinesis. Like Type II, facial disfigurement with synkinesis is the major reason to seek for treatment. Patients in Type III demonstrate a greater ambition to accept aggressive procedures to correct their disfigurements [Figure 2B].

\section{Type IV: poor smile and mild synkinesis}

Type IV PPFS was seen in less than $1 \%$ of the population ${ }^{[26]}$. In Type IV PPFS, patients have near complete facial paralysis with mild synkinesis. Lagophthalmos is always noted. The patient's chief complaints for requesting a treatment are related to the paralytic face, less on the synkinesis.

A retrospective review was performed between January 1985 and August 2020 (35 year period, Figure 1). A total of 430 facial palsy patients were treated by gracilis-FFMT for smile reconstruction. Of these, 103 (24 \%) were PPFS patients. The rate of treating PPFS patients with a surgical approach increased from $15 \%$ prior to $2012^{[26]}$ up to $24 \%$.

After exclusion of 9 patients in 2020, a total of 94 PPFS patients were selected and enrolled in this study. They all had at least one year of postoperative follow-up. Patient characteristics including gender, age at the time of surgery, etiology, affected side, type of PPFS, and FFMT neurotized by different motor neurotizers (CFNG, cross-facial nerve graft; XI, spinal accessory nerve; V3, masseter nerve) were recorded [Table 2].

In the selected 94 PPFS patients, 50 patients were Type II, and 44 patients were Type III. They all underwent an aggressive surgical intervention, which included combined myectomy \& neurectomy, and smile reconstruction with gracilis FFMT. 
Table 2. PPFS Patient Demographics

\begin{tabular}{llll}
\hline Total No. patients & $\mathbf{1 0 0}$ & Pts \\
\hline Mean age (range), years & $32.2(6-73)$ & $<15 \mathrm{Y} / \mathrm{O}$, & 6 \\
& & $15-49$ & 77 \\
& & $>50$ & 17 \\
Gender (Male/Female) & $36: 64$ & & \\
Affected side (right : left) & $53: 47$ & & \\
Etiology & & 35 \\
Bell's palsy & & 22 \\
Trauma & & 19 \\
Infection & & 18 \\
After tumor resection & & 4 \\
Facial nerve injury and repair & & 1 \\
Other (brain infarction) & & \\
\hline
\end{tabular}

\section{Myectomy \& neurectomy}

Extensive removal of the synkinetic muscles (cheek, nose and neck) and triggered nerves (zygomatic, buccal and colli facial nerve branches) on the affected face.

This was performed during the first stage of XI-gracilis and V3-gracilis, or the 2nd stage of CFNG-gracilis. Four incisions were made on the affected side of the face [Figure 3A]. The first incision was on the white line of the upper lip. The incision went directly and vertically down to the above-mucosa layer, leaving the orbicularis oris muscle on the lip side. If the CFNG was performed in the first stage, the short CFNG was identified, mobilized, and protected in the lip wound.

Three to four 4-0 Dexon anchoring sutures were placed behind the orbicularis oris muscle for a later gracilis aponeurosis attachment. The second incision was $1 \mathrm{~cm}$ in length on the white line of the lower lip for lower lip suspension with gracilis tail. The third incision was a long preauricular incision from the temporal hair and down to the mandibular body for a subcutaneous face-lift. Under the elevated skin flap, a trapezoid with four sides was drawn from the zygomatic arch down to the upper lip [Figure 3B]. Dissection was performed along the surface of the masseter fascia and muscle down to the inferior edge of the drawn trapezoid area. All tissues in this trapezoid-marked area, including the upper lip elevators (zygomatic muscles, levator labii superioris, buccinator, caninus), some of the nose muscles (nasalis), and zygomatic and buccal facial nerve branches were removed en bloc [Figure 3C]. Parotid duct (Stensen's duct) was protected and preserved to avoid injury and saliva leak. An assistant typically inserted a finger into the mouth to guide soft tissue removal and avoid penetration into the oral mucosa. The buccal fat pad was routinely removed subtotally to decrease the volume and to avoid its herniation in the future. A subcutaneous space was created [Figure 3D]. A $3-\mathrm{cm}$ transverse incision down to the zygomatic arch bone was made. The incision could be extended medially to the zygomatic body, and laterally but not beyond the line of the pathway of the temporal facial nerve branch. The temporal facial nerve branch was protected to avoid lagophthalmos. Three to four 3-0 Dexon stitches were anchored on the suprazygomatic margin periosteum for a later gracilis muscle attachment. Corrugator muscle, if preoperatively noted to have hypertrophy, was removed through another small suprabrow incision. On the mandible wound, the wide insertion of the platysma on the mandible was totally exposed and dissected down to its insertion on the clavicle. Because of its breadth, a 4th supraclavicular access incision was always required. Total removal of the platysma was required because it was a major synkinetic muscle [Figure $3 \mathrm{~A}$ and $\mathrm{C}$ ]. Under the platysma, the mandibular facial nerve branch crossing the facial artery and vein was found and protected to preserve lower lip movement. The facial vessels were dissected and prepared for later vessel anastomoses to the transferred muscle's vessels. A suction drain was always placed in the neck wound because oozing was much more than the surgery for PFP. 

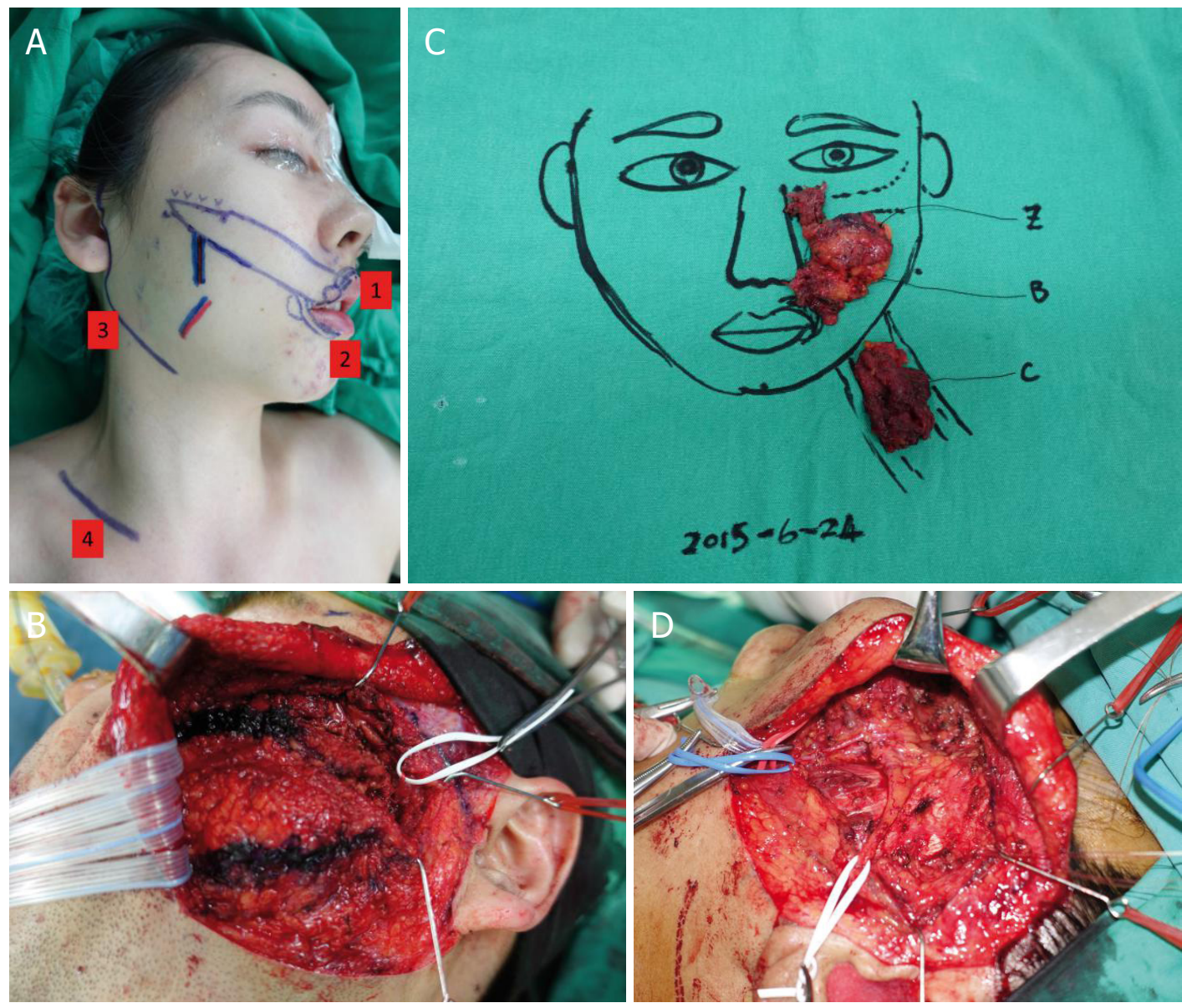

Figure 3. Regularly there are four incision wounds (see Text) (A); a trapezoid with four sides is drawn from the zygomatic arch down to the upper lip (B); picture shows the removed muscles and nerves (C); picture shows the midface creative space, leaving the Stensen's duct, masseter muscle and facial vessels intact (D)

Smile reconstruction with gracilis FFMT

Dissection and harvest of the gracilis from the contralateral thigh was performed simultaneously with the surgical ablation described above. The indication and surgical details for three methods have been reported in prior publications ${ }^{[10,11]}$.

\section{Postoperative care and rehabilitation protocol after FFMT}

After ablative and reconstruction surgery, the midface became nearly paralytic. Postoperative care and rehabilitation were principally the same as CFNG-, XI- and V3-gracilis transplantation for smile reconstruction, including massage, electric stimulation, and "Induction Exercise" ${ }^{\text {"[10,11] }}$.

Two extra rehabilitation procedures, which were proposed and advocated in recent years, were followed: (1) "upper lip stretching exercise" [Video 1] on the reconstructed side was usually performed 6 months postoperatively, when the gracilis muscle started to move (M1). The "stretching exercise" was performed daily and continued forever to avoid potential lip contracture; and (2) smiling training in front of the mirror was performed once the gracilis muscle function reached to M2 (two teeth visible). This usually occurred one year after FFMT. Smiling on the reconstructed side is strictly triggered by the healthy side and avoided by the reconstructive side to avoid recurrent synkinesis. 


\section{Outcome assessment}

Postoperatively, all patients were evaluated clinically to confirm the function of FFMT without EMG. All patients were closely followed every 2 months for at least one year. Patients were photographed and videotaped during pre-and postoperative visits in a standardized fashion, including face at repose, face at smile (mild, moderate and maximal smile), and degree of residual synkinesis of the eye, mouth and neck. Outcomes were assessed in terms of success of decreasing synkinesis and achieving facial symmetry at rest and smiling, which were recorded in the special chart.

Six methods were utilized for outcome assessment and included: (1) smile Excursion Score (score 0 to 4) to check muscle strength based on the exposure of denture during maximal smile [Supplementary digital content 2]; (2) cortical Adaptation Staging (stage I to V) to check for smile movement whether it is controllable, and spontaneous. [Supplementary digital content 3]; (3) hadlock Smile Measurement of Improvement in Lip Excursion Scale to quantitatively measure paralyzed lip excursion pre- and postoperatively ${ }^{[11]}$; (4) terzis Functional and Aesthetic Grading for general evaluation (Grade I-V) ${ }^{[11]}$. Grade 4 (good) and 5 (excellent) were considered acceptable; (5) Synkinesis Grading System (from none to $3+$ ); and (6) patient's questionnaire and "Satisfaction Score (1-5)". Scores $\geq 3$ were considered acceptable. Patients were reviewed and graded by five independent reviewers (two attendings, two micro-fellows and one assistant) based on photos and videos.

\section{Statistical analysis}

Statistical analysis was performed using SPSS 22.0 software (SPSS, Inc., Chicago, Ill). Data were presented with mean and \pm standard deviation. Mann-Whitney $U$ test was used for comparison between two independent samples and Kruskal-Wallis test for more than two independent groups. Nonparametric test was applied for unequal patient numbers in different groups with skewed distributions. $\mathrm{P}$ values less than 0.05 were considered statistically significant.

\section{RESULTS}

Young adults $(79 \%)$ and female patients (63\%) were dominant in the study population, showing their great ambition for receiving treatment.

The mean Smile Excursion Score in all three groups were significantly improved from $0.550 \pm 0.076$ preoperatively to $3.160 \pm 0.093$ postoperatively (> 3 years) in CFNG-gracilis. $0.325 \pm 0.038$ to $3.835 \pm 0.060$ in XI-gracilis, and $0.132 \pm 0.047$ to $3.600 \pm 0.245$ in V3-gracilis $(P<0.05)$. Cortical Adaptation Stage were at Stage IV or V in CFNG-gracilis and XI-gracilis groups, but at Stage III in V3-gracilis. Mean Hadlock Lip Excursion Scale showed significant improvement in lip excursion (mean 14.12 mm, range 12.66-16.26) compared with the preoperative lip excursion in CFNG-gracilis, XI- (mean $7.4 \mathrm{~mm}$, range 3.75-13.68) and V3-gracilis (mean $9.25 \mathrm{~mm}$, range 1.81-18.05) $(P=0.007)$. For patients with more than 3 years of follow-up postoperatively, the Terzis Functional and Aesthetic Grading System also showed a significant improvement from $1.367 \pm 0.049$ preoperatively to $4.180 \pm 0.146$ in CFNG-gracilis, $1.347 \pm 0.053$ to $4.439 \pm 0.202$ in XIgracilis, and $1.037 \pm 0.023$ to $4.100 \pm 0.245$ in V3-gracilis. The synkinesis grading system was significantly improved in all groups, from moderate $(2+)$ or severe $(3+)$ down to mild $(1+)$ or even no synkinesis (subclinical) $(P<0.001)$. The Satisfactory score was $86 \%$ in CFNG-gracilis, $90 \%$ in XI-gracilis and $67 \%$ in V3-gracilis group with a raw score greater than or equal to 3. No patient regretted having the complex surgical procedures.

The 90 (96\%) patients felt that their synkinesis was significantly decreased after surgery and had no need for a BTX-A injection. The improvement of synkinesis started on the first day postoperatively and continued to improve throughout the long-term follow up and smiling training. The most improved sites were the eye 


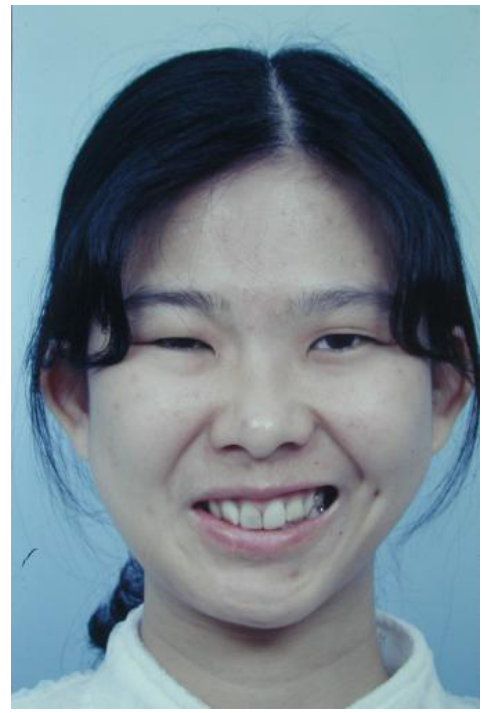

Figure 4. A $24 \mathrm{Y} / \mathrm{O}$ girl (the same patient as in Figure 2A) had right PPFS type II for 2 years due to a car accident. Preoperative photos showed right face with acceptable smile but moderate synkinesis. She received the aggressive surgical intervention. She showed good smile and symmetric eye with no more synkinesis three years after CFNG-gracilis and blepharoplasty. CFNG: cross-facial nerve graft; PPFS: postparalytic facial synkinesis

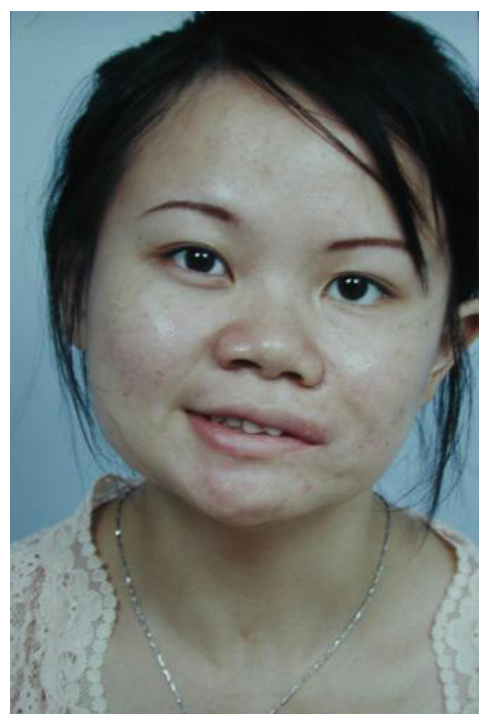

Figure 5. A $20 \mathrm{Y} / \mathrm{O}$ girl had right PPFS type III due to chronic otitis media with cholesteatoma resection in her childhood. Preoperative photo shows right face with poor smile and severe synkinesis (the same patient as Figure 2B). She received the aggressive surgical intervention. She showed good and symmetric smile with no more synkinesis two years after CFNG-gracilis. CFNG: cross-facial nerve graft; PPFS: postparalytic facial synkinesis

and neck areas with absence of eye narrowing and neck bands. Many patients also showed disappearance of synkinesis in the clinic follow-up. The synkinesis became subclinical.

Representative cases are shown in Figure 4 (postoperatively, the same patient as Figure 2A), Figure 5 (postoperatively, the same patient as Figure 2B), Figure 6 (preoperatively) and Video 2 (result), Figure 7 (preoperatively) and Video 3 (result), Figure 8 (preoperatively) and Video 4 (result), and Figure 9 (preoperatively) and Video 5 (result). 


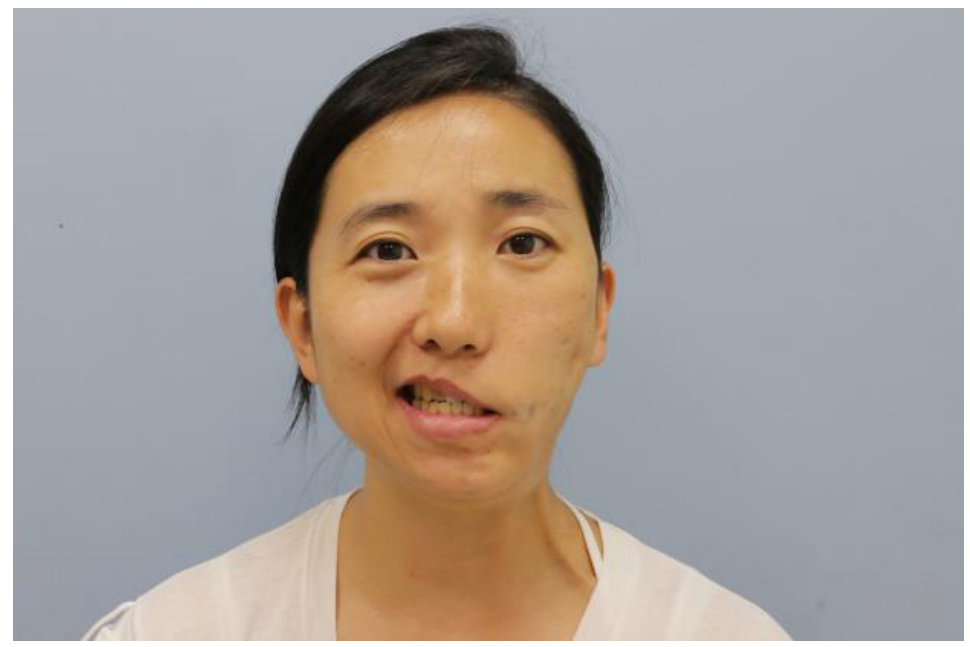

Figure 6. A 38 Y/O lady developed left PPFS type III for 17 years following multiple operations for left cheek hemangioma (6 operations including excision and intralesional laser surgery, and 9 sclerotic injections). She received the aggressive surgical intervention. PPFS: postparalytic facial synkinesis

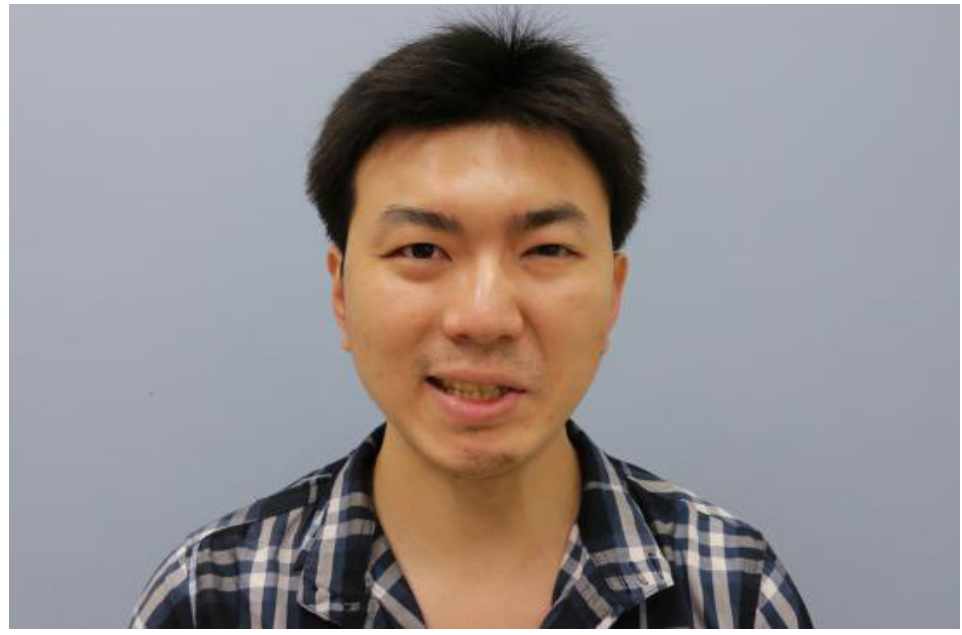

Figure 7. A 31 Y/O male developed left PPFS type III for 27 years following a car accident at the age of 3 Y/O. He received the aggressive surgical intervention. PPFS: postparalytic facial synkinesis

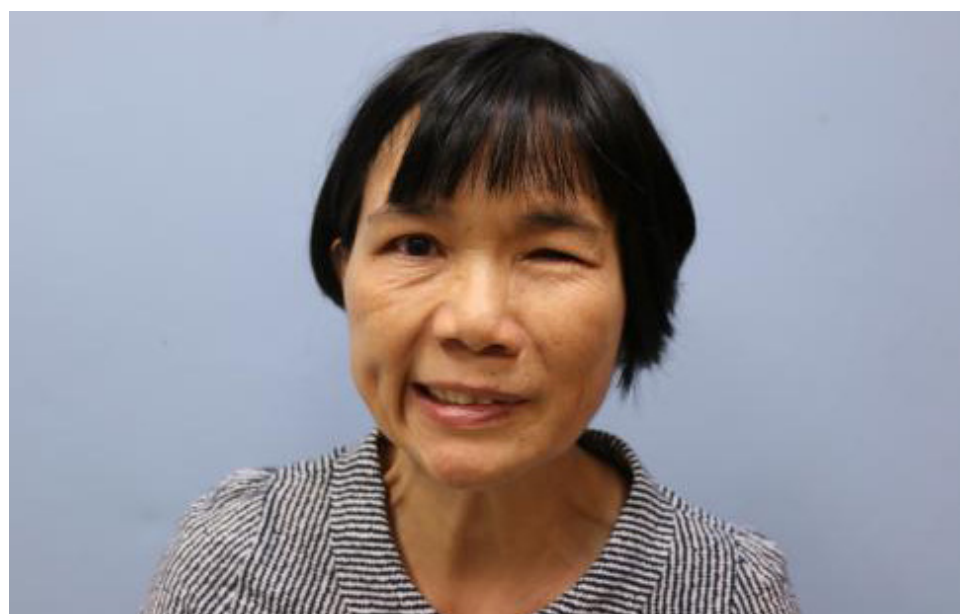

Figure 8. A $56 \mathrm{Y} / \mathrm{O}$ male developed left PPFS type III due to Herpes zoster infection for 6 years. She had a decompression surgery by an ENT surgeon. She received the aggressive surgical intervention. PPFS: postparalytic facial synkinesis 


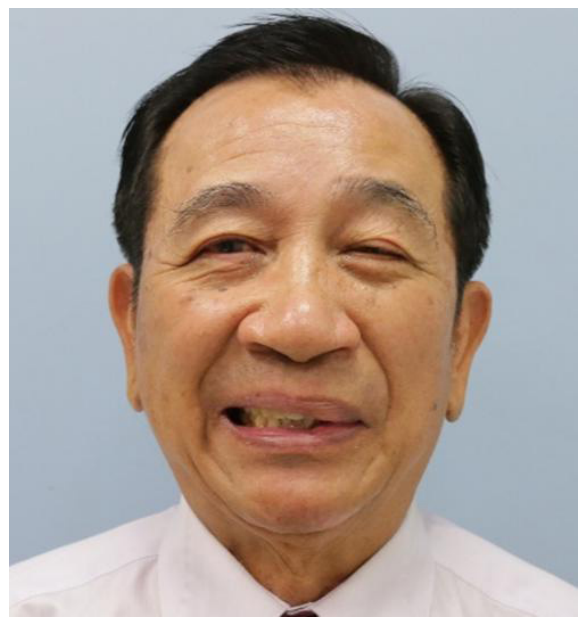

Figure 9. A 72 Y/O male developed left PPFS Type III due to left Bell's palsy for 30+ years. He received the aggressive surgical intervention. PPFS: postparalytic facial synkinesis
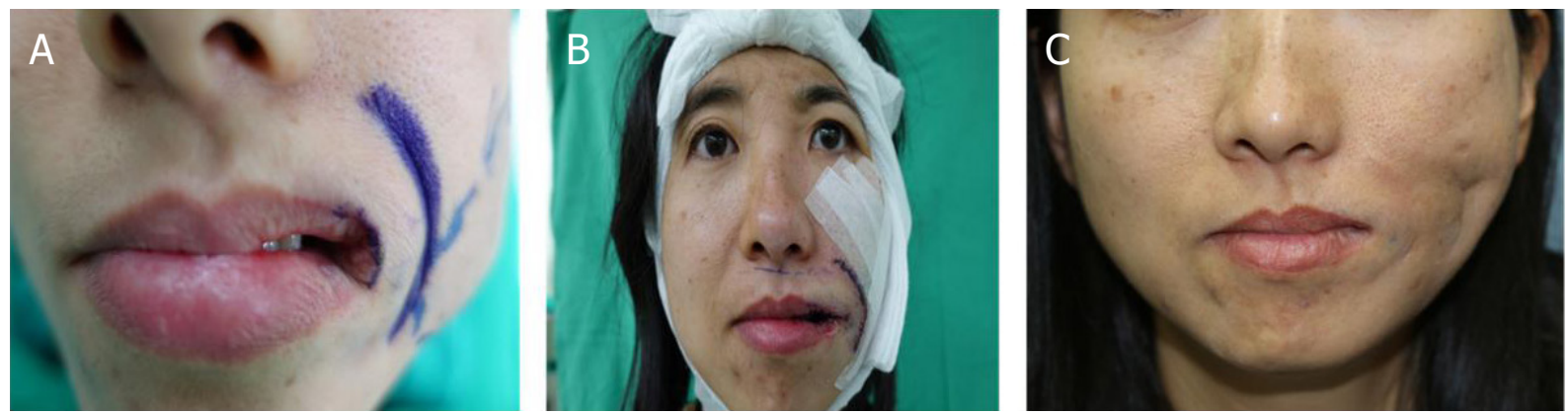

Figure 10. A 38Y/O, after multiple hemangioma treatments, developed PPFS Type II: (A) contracture of the left upper lip and deep nasolabial fold $1.5 \mathrm{Y}$ after CFNG-gracilis; (B)immediate result after revision surgery with release of contracture and dermofat graft; (C) one year after revision surgery. PPFS: postparalytic facial synkinesis

\section{Complications}

Acute complications such as flap reopen, flap loss, hematoma, saliva leak or wound dehiscence were rare. The most significant complication was a high revision rate (approximately 53\%) in the long-term followup after FFMT reconstruction. Major secondary deformities [Table 3] included: (1) deep fold on the upper lip and/or mouth angle [Figure 10A]; (2) wide lip deformity [Figure 11A]; (3) deep nasolabial fold; and (4) bulky cheek while smiling.

A deep fold on the upper lip and/or mouth angle, and a dominant nasolabial fold were the most common complications, and occurred in 44 patients (47\%). Gracilis contracture and/or scars were considered the causes. Revision surgery, if needed, was performed one year after the FFMT. Revision for the contracture included surgical release of the contracture and dermofat graft to fill the created space [Figure $10 \mathrm{~B}$ and C]. The deep nasolabial fold was corrected by rigotomy and fat grafts, or by tensor fascia lata suspension. Wide vermilion exposure was corrected by a thinning procedure on the upper lip [Figure $11 \mathrm{~B}$ and $\mathrm{C}$ ]. Bulky smile was corrected by an upward advancement of the gracilis attachment. Other procedures included release of the gracilis tail attachment for tight lower lip, fat grafts for depression or uneven surface. Aesthetic surgeries including browlift, midface lift, fat grafts, blepharoplasty and tensor fascia lata suspension were often performed to enhance the result. BTX-A injection was usually not required. 

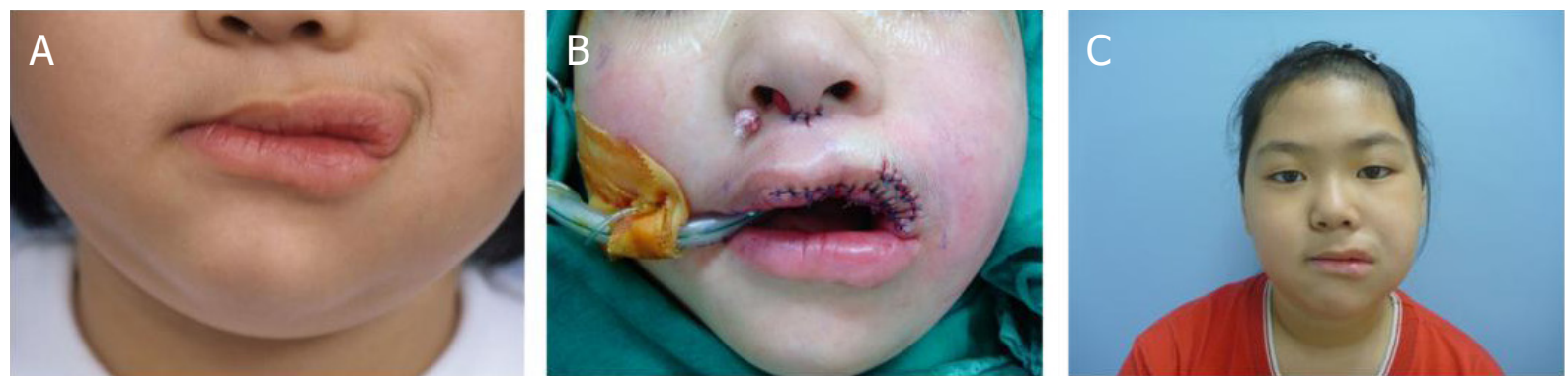

Figure 11. A $5 \mathrm{Y} / \mathrm{O}$ girl had left facial palsy since birth: (A)she developed contracture of the left upper lip and too wide vermilion exposure 1Y6M after CFNG-gracilis, (B) immediate result after release of contracture and thinning procedure. (C) $3 Y 6 \mathrm{M}$ after revision surgery. CFNG: cross-facial nerve graft

Four patients (4\%) required BTX-A injection due to a recurrent lip contracture from the underling gracilis after revision surgery. One patient who failed the CFNG-innervated gracilis received a masseter-innervated 2nd gracilis for reconstruction one year after the 1st gracilis removal and showed improved results.

\section{DISCUSSION}

\section{Philosophy of PPFS treatment}

Aberrant reinnervation may occur in motor and sensory distributions following peripheral nerve degeneration and regeneration. Motor recovery with aberrant reinnervation accompanies synkinesis (or co-contraction), which especially occurs in PPFS and obstetrical brachial plexus palsy ${ }^{[27,28]}$. Only aggressive surgical intervention can effectively resolve the problem, including muscle transfers and FFMT ${ }^{[26-28]}$.

Once a synkinesis, always a synkinesis. Treatment of PPFS is a warto patients, surgeons and rehabilitation therapists, just like a scar is a war. It requires team work and more patience. The treatment should include a good preoperative explanation, an aggressive surgical intervention, postoperative long-term rehabilitation and close clinical follow-up, and the abilities to correct any potential deformities. Our results were longlasting and $96 \%$ of treated patients did not require an additional BTN-A injection.

\section{Surgical procedure for PPFS and PFP}

Surgical procedure for PPFS is quite different from PFP: (1) Soft tissue removal: For incomplete PFP, only soft tissues above the facial nerve branches between the zygoma bone and upper lip are removed to create a shallow trough for a muscle inset. For complete PFP, the soft tissues above the masseter muscle are removed to create a bigger space. For PPFS, the soft tissue removal is similar to complete PFP, but is more extensive and includes the midface facial mimetic muscles, neck platysma, corrugator and some nasal muscles. But temporal and mandibular facial nerve branches should be preserved to prevent lagophthalmos and lower lip asymmetry; and (2) the gracilis FFMT is trimmed longer than when used in PFP to avoid subsequent contracture.

\section{Higher revision rates}

There are several potential reasons: (1) the harvested gracilis might be too short; (2) reversal of the gracilis puts the aponeurosis into the movable upper lip; and (3) the residual synkinesis. Although the midface mimetic muscles and the zygomatic and buccal facial nerve branches are extensively removed, there are still residual muscles in the perioral region, around 30\%. Those residual muscles are still functionally innervated by the superior temporal and inferior mandibular facial nerve branches. A refined gracilis harvest and inset, and intensive postoperative rehabilitation are ongoing in recent years to decrease the revision rates. 
Table 3. Deformities following FFMT

\begin{tabular}{|c|c|c|c|}
\hline Location & Deformity & Surgical Correction & Incidence (pts) \\
\hline \multirow[t]{7}{*}{ Upper lip } & \multirow{6}{*}{$\begin{array}{l}\text { Contracture of mouth angle or upper lip with } \\
\text { abnormal deep fold formation }\end{array}$} & 1. release of contracture and dermafat graft & 14 \\
\hline & & 2. TFL cheek skin suspension & 9 \\
\hline & & 3. $\mathrm{V}-\mathrm{Y}$ plasty or Z-plasty & 10 \\
\hline & & 4. botox & 4 \\
\hline & & 5. selective neurectomy of the affected facial nerve & 2 \\
\hline & & 6. lip adhesion (2 stages) & 5 \\
\hline & Wide vermilion & Thinning procedure & 5 \\
\hline \multirow[t]{5}{*}{ Cheek } & \multirow[t]{2}{*}{ Bulkiness } & 1. debulking & 5 \\
\hline & & 2. gracilis upward advancement & 2 \\
\hline & Uneven surface & Rigotomy and fat grafts & 18 \\
\hline & Deep nasolabial fold/Marrionette line & $\begin{array}{l}\text { 1. rigotomy and fat graft } \\
\text { 2. TFL cheek skin suspension } \\
\text { 3. V-Y plasty or Z-plasty }\end{array}$ & Overlapped \\
\hline & Abnormal facial wrinkles & Rigotomy and fat grafts & Overlapped \\
\hline \multirow[t]{6}{*}{ Eyelid } & Corrugator muscle hypertrophy & Corrugator resection & 9 \\
\hline & Ptosis & Browlift & 2 \\
\hline & \multirow[t]{3}{*}{ Lagophthalmos } & 1. lateral tarsorrhaphy & 5 \\
\hline & & 2. temporalis muscle transfer & 1 \\
\hline & & 3. FFMT & 5 \\
\hline & Asymmetry & Double eyelid formation & 8 \\
\hline \multirow[t]{6}{*}{ Lower lip } & \multirow[t]{2}{*}{ Asymmetry } & 1. wedge resection of the lower lip & 2 \\
\hline & & 2. myectomy of the healthy side depressors & 6 \\
\hline & \multirow[t]{3}{*}{ Drooling/dimpling } & 1. wedge resection & 2 \\
\hline & & 2. plantaris tendon suspension & 1 \\
\hline & & 3. dermofat graft & 1 \\
\hline & Tight band & Release of aponeurosis or plantaris tendon & 2 \\
\hline Others & Hypertrophic scars & Scar revision & 1 \\
\hline
\end{tabular}

FFMT, functioning free muscle transplantation

Table 4. Advantages and disadvantages between Selective Neurectomy and Aggressive Surgical Procedures

\begin{tabular}{|c|c|c|}
\hline & Selective Neurectomy & Aggressive Surgical Procedures \\
\hline Representative & Azizzadeh B & Chuang DCC \\
\hline Advantages & $\begin{array}{l}\text { 1. Simple operation (neurectomy) } \\
\text { 2. Quick surgery ( } 2-3 \text { hrs) } \\
\text { 3. Less scars } \\
\text { 4. Less facial disfigures postoperatively }\end{array}$ & $\begin{array}{l}\text { 1. Technique: complex but straight forward } \\
\text { 2. Usually no need of BTX-A } \\
\text { 3. Usually no need for simultaneous rhytidectomy } \\
\text { 4. } 2^{\text {nd }} \text { deformity can be easily corrected by revision } \\
\text { surgery (usually performed at one year postoperatively) } \\
\text { 5. Results: predictable and long-lasting }\end{array}$ \\
\hline Disadvantages & $\begin{array}{l}\text { 1.Technique: difficult in decision making, and } \\
\text { unpredictable results } \\
\text { 2. High need for adjuvant surgery such as rhytidectomy, } \\
\text { fat grafting } \\
\text { 3. Continues need BTX-A treatment } \\
\text { 4.Timing of revision surgery: uncertain (immediately or } \\
\text { days later?) }\end{array}$ & $\begin{array}{l}\text { 1.Complex operation (neurectomy + myectomy + } \\
\text { reconstruction) } \\
\text { 2. Long surgery ( } 6-10 \text { hrs) } \\
\text { 3. Need micro-neural- vascular anastomoses } \\
\text { 4.More operative scars } \\
\text { 5. High rates of revision surgery }\end{array}$ \\
\hline
\end{tabular}

\section{Comparison between selective neurectomy and aggressive surgical intervention (combined myectomy \& neurectomy and FFMT)}

Surgical neurectomy has been used for more than 50 years to treat hemifacial spasm and blepharospasm ${ }^{[19-22]}$. Both conditions are primary facial spasms caused by vascular compression of the facial nerve in the brain stem ${ }^{[29,30]}$. PPFS is secondary facial spasm. Azizzadeh et al. ${ }^{[23]}$ applied selective neurectomy, called "modified midface selective neurectomy" to treat a large series of 65 PPFS patients between 2013 and 2017, and reported the data in 2019. Comparison of advantages and disadvantages between Azizzadeh's and Chuang's technique are made in Table 4. 


\section{Why do patients accept complex surgery with a higher revision rate?}

PPFS surgery is reconstructive, not cosmetic. The treatment requires team work, including comprehensive preoperative explanation, postoperative long-term rehabilitation and close clinical follow-up, and surgical skills to treat the second deformity. The results for the Type II and III PPFS treatment is effective and longlasting. They were satisfied with the results despite a high revision rate.

In conclusion, treatment of PPFS is primarily reconstructive, not aesthetic. Combined myectomy \& neurectomy and FFMT are aggressive. The patients, Type II and III PPFS, accept it because of the promising and long-lasting results, despite a high revision rate. Refined techniques to decrease the revision rates are needed.

\section{DECLARATIONS}

\section{Authors' contributions}

The author contributed solely to the article.

\section{Availability of Data and Materials}

Not applicable.

\section{Financial Support and Sponsorship}

None.

\section{Conflict of interest}

All authors declared that there are no conflicts of interest.

\section{Ethical Approval and Consent to Participate}

The study had Department approval and conforms to the Helsinki Declaration with Institutions Review Board (IRB).

\section{Consent for publication}

Written consent for publication was obtained for all images and video.

\section{Copyright}

(c) The Author(s) 2021.

\section{REFERENCES}

1. Moran CJ, Neely JG. Patterns of facial nerve synkinesis. Laryngoscope 1996;106:1491-6.

2. Celik M, Forta H, Vural C. The development of synkinesis after facial nerve paralysis. Eur Neurol 2000;43:147-51.

3. Valls-Solé J, Montero J. Movement disorders in patients with peripheral facial palsy. Mov Disord 2003;18:1424-35.

4. Oge AE, Yayla V, Demir GA, Eraksoy M. Excitability of facial nucleus and related brain-stem reflexes in hemifacial spasm, post-facial palsy synkinesis and facial myokymia. Clin Neurophysiol 2005;116:1542-54.

5. Beurskens CH, Oosterhof J, Nijhuis-van der Sanden MW. Frequency and location of synkineses in patients with peripheral facial nerve paresis. Otol Neurotol 2010;31:671-5.

6. Terzis JK, Noah ME. Analysis of 100 cases of free-muscle transplantation for facial paralysis. Plast Reconstr Surg 1997;99:1905-21.

7. Manktelow RT. Free muscle transplantation for facial paralysis. Clin Plast Surg 1984;11:215-20.

8. Zuker RM, Manktelow RT, Hussain G. Facial paralysis. In Mathes S, Hentz V editors. Plastic Surgery. Philadelphia: Elsevier Inc., 2006, pp.883-916.

9. Guelinckx PJ, Sinsel NK. Muscle transplantation for reconstruction of a smile after facial paralysis past, present, and future. Microsurgery 1996;17:391-401.

10. Chuang DC. Technique evolution for facial paralysis reconstruction using functioning free muscle transplantation-experience of Chang Gung Memorial Hospital. Clinics in Plastic Surgery 2002;29:449-59.

11. Chuang DC, Lu JC, Chang TN, Hamdi Sakarya A. Using the "sugarcane chewing" concept as the directionality of motor neurotizer selection for facial paralysis reconstruction: Chang Gung experiences. Plast Reconstr Surg 2019;144:252e-63e. 
12. VanSwearingen JM, Brach JS. Changes in facial movement and synkinesis with facial neuromuscular reeducation. Plast Reconstr Surg 2003;111:2370-5

13. Brach JS, VanSwearingen JM, Lenert J, Johnson PC. Facial neuromuscular retraining for oral synkinesis. Plast Reconstr Surg 1997;99:1922-31; discussion 1932-3.

14. Diels HJ, Combs D. Neuromuscular retraining for facial paralysis. Otolaryngol Clin North Am 1997;30:727-43.

15. Putterman AM. Botulinum toxin injections in the treatment of seventh nerve misdirection. American Journal of Ophthalmology 1990;110:205-6.

16. Rogers CR, Schmidt KL, VanSwearingen JM, et al. Automated facial image analysis: detecting improvement in abnormal facial movement after treatment with botulinum toxin A. Ann Plast Surg 2007;58:39-47.

17. Armstrong MW, Mountain RE, Murray JA. Treatment of facial synkinesis and facial asymmetry with botulinum toxin type A following facial nerve palsy. Clin Otolaryngol Allied Sci 1996;21:15-20.

18. Terzis JK, Karypidis D. Therapeutic strategies in post-facial paralysis synkinesis in adult patients. Plast Reconstr Surg 2012;129:925e-39e.

19. Coleman CC. Surgical treatment of facial spasm. Ann Surg 1937;105:647-57.

20. Marino H, Alurralde A. Spastic facial palsy-peripheral selective neurotomy. Br J Plast Surg 1950;3:56-9.

21. Fisch U, Esslen E. The surgical treatment of facial hyperkinesia. Arch Otolaryngol 1972;95:400-5.

22. Hohman MH, Lee LN, Hadlock TA. Two-step highly selective neurectomy for refractory periocular synkinesis. Laryngoscope 2013;123:1385-8

23. Azizzadeh B, Irvine LE, Diels J, et al. Modified selective neurectomy for the treatment of post-facial paralysis synkinesis. Plast Reconstr Surg 2019;143:1483-96.

24. Guerrissi JO. Selective myectomy for postparetic facial synkinesis. Plast Reconstr Surg 1991;87:459-66.

25. Henstrom DK, Malo JS, Cheney ML, Hadlock TA. Platysmectomy: an effective intervention for facial synkinesis and hypertonicity. Arch Facial Plast Surg 2011;13:239-43.

26. Chuang DC, Chang TN, Lu JC. Postparalysis facial synkinesis: clinical classification and surgical strategies. Plast Reconstr Surg Glob Open 2015;3:e320.

27. Chuang DC, Ma HS, Wei FC. A new strategy of muscle transposition for treatment of shoulder deformity caused by obstetric brachial plexus palsy. Plast Reconstr Surg 1998;101:686-94.

28. Chuang DC, Hattori Y, Ma And HS, Chen HC. The reconstructive strategy for improving elbow function in late obstetric brachial plexus palsy. Plast Reconstr Surg 2002;109:116-26; discussion 127-9.

29. Colosimo C, Bologna M, Lamberti S, et al. A comparative study of primary and secondary hemifacial spasm. Arch Neurol 2006;63:441-4.

30. Valls-Solé J. Electrodiagnostic studies of the facial nerve in peripheral facial palsy and hemifacial spasm. Muscle Nerve 2007;36:14-20. 